\title{
A Microarray Study of Middle Cerebral Occlusion Rat Brain with Acupuncture Intervention
}

\author{
Chao Zhang, ${ }^{1}$ Yan Wen, ${ }^{1}$ Xiaonong Fan, ${ }^{2,3,4,5}$ Sha Yang, ${ }^{2}$ Guang Tian, \\ Xueyi Zhou, ${ }^{6}$ Yaqiong Chen, ${ }^{2}$ and Zhihong Meng ${ }^{1}$ \\ ${ }^{1}$ Department of Acupuncture and Moxibustion, First Teaching Hospital of Tianjin University of Traditional Chinese Medicine, \\ Tianjin 300193, China \\ ${ }^{2}$ Acupuncture and Moxibustion Research Institute of Tianjin University of Traditional Chinese Medicine, Tianjin, China \\ ${ }^{3}$ Key Laboratory of Cerebropathy Acupuncture Therapy of State Administration of Traditional Chinese Medicine, \\ Tianjin 300193, China \\ ${ }^{4}$ Tianjin Key Laboratory of Acupuncture \& Moxibustion Science, Tianjin 300193, China \\ ${ }^{5}$ Three-Level Laboratory of Acupuncture Dose-Effect Relationship, State Administration of Traditional Chinese Medicine, \\ Tianjin 300193, China \\ ${ }^{6}$ Post-Graduate School, Tianjin University of Traditional Chinese Medicine, Tianjin 300193, China
}

Correspondence should be addressed to Zhihong Meng; tjmzh86@163.com

Received 19 November 2014; Revised 24 December 2014; Accepted 5 February 2015

Academic Editor: Adair Santos

Copyright (C) 2015 Chao Zhang et al. This is an open access article distributed under the Creative Commons Attribution License, which permits unrestricted use, distribution, and reproduction in any medium, provided the original work is properly cited.

\begin{abstract}
Microarray analysis was used to investigate the changes of gene expression of ischemic stroke and acupuncture intervention in middle cerebral artery occlusion (MCAo) rat brain. Results showed that acupuncture intervention had a remarkable improvement in neural deficit score, cerebral blood flow, and cerebral infarction volume of MCAo rats. Microarray analysis showed that a total of 627 different expression genes were regulated in ischemic stroke. 417 genes were upregulated and 210 genes were downregulated. A total of 361 different expression genes were regulated after acupuncture intervention. Three genes were upregulated and 358 genes were downregulated. The expression of novel genes after acupuncture intervention, including Tph1 and Olr883, was further analyzed by Real-Time Quantitative Polymerase Chain Reaction (RT-PCR). Upregulation of Tph1 and downregulation of Olr883 indicated that the therapeutic effect of acupuncture for ischemic stroke may be closely related to the suppression of poststroke depression and regulation of olfactory transduction. In conclusion, the present study may enrich our understanding of the multiple pathological process of ischemic brain injury and indicate possible mechanisms of acupuncture on ischemic stroke.
\end{abstract}

\section{Introduction}

Stroke is still a worldwide disease today which causes millions of deaths and more numbers of physical disabilities every year $[1,2]$. It is also the leading cause of death and long-term disability in China, where the annual stroke mortality rate is approximately 1.6 million. There are over 7 million stroke survivors in the country with $70 \%$ of them being ischemic $[3,4]$. The complexity of pathological changes involved in the process of ischemic stroke, worsened by the narrow treatment window (3-6 hs) of rt-PA therapy and the risk of hemorrhage of antiplatelet therapy and anticoagulation therapy [5], has led to the unoptimistic situation of the treatment of ischemic stroke. Sequela syndromes are commonly seen in ischemic stroke patients; thus physical training (PT) and other complementary therapy are increasingly used in ischemic stroke patients.

Acupuncture is a traditional Chinese medical therapy which has been proved effective in the treatment of ischemic stroke [6-9]. Researches have found that acupuncture can effectively improve the motor function of ischemic stroke patients [10], possibly due to the improvement of cerebral circulation [11-13], but the underlying biological mechanisms of acupuncture effects are still poorly understood and need to be further explored. 


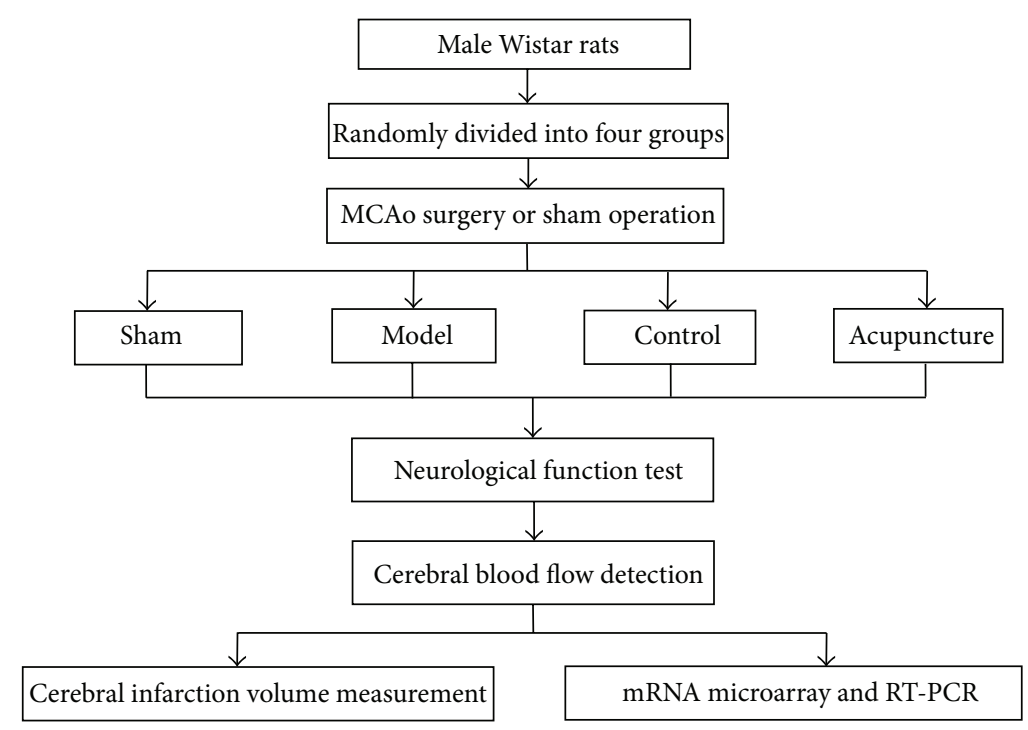

FIGURE 1: Schematic diagram for methodologies: a total of 72 adult male Wistar rats were randomly divided into the sham, model, control, and acupuncture groups. Rats in the model group underwent the measurements of neurological function, cerebral blood flow (CBF), and cerebral infarction volume immediately after they regained consciousness from anesthesia while rats in other groups received the measurements 60 hours later. Six rats were randomly selected from each group for mRNA microarray and RT-PCR test and 12 others for cerebral infarction volume measurement.

Microarray research has been a hot topic since the last decade. Gene-chips provide the means to measure simultaneously where and when thousands of genes are expressed [14]. This technology allows detection and quantification of the differential expression of thousands of genes simultaneously in a single experiment. This technology has helped illuminate mechanisms of disease and identify disease subphenotypes, predict disease progression, assign function to previously unannotated genes, and group genes into functional pathways [15]. Thus, many researches have tried to explore the secret of stroke by using gene-chip technology [16-21] and a lot of biological processes have been found to be related to the pathological injury of ischemic stroke such as inflammation [22], apoptosis [20], and excitotoxicity [23]. So we assume that the effect of acupuncture on ischemic stroke may be acquired from the regulation of these biological processes. In the present study, gene-chip technology was used to analyze the gene expression in ischemic stroke and acupuncture intervention to find out how acupuncture regulates the gene expression of ischemia brain tissues in the model of MCAo rats and thus to reveal possible biological mechanisms of acupuncture on ischemic stroke.

\section{Experimental Procedures}

2.1. Experimental Groups and MCAo Model. Male Wistar rats weighing 250-300 g, aged 3 months, were selected from the Laboratory Animal Center of People's Liberation Army Academy of Military Medical Sciences, Beijing, China [License no. SCXK-(Army) 2012-0004]. Animals were acclimated to the animal quarters at least for 3 days before the experiment and were provided with a standard laboratory diet and water ad libitum. Animal treatments were performed in a manner to minimize pain or discomfort in accordance with the Guidance Suggestions for the Care and Use of Laboratory Animals, formulated by the Ministry of Science and Technology of China. Rats were randomly divided into 4 groups including model, sham, control, and acupuncture. The minimum number of animals $(n=18)$ was used for each group. Rats in the model, control, and acupuncture groups were subjected to MCAo surgery. The difference between the model and control groups was that rats in the model group underwent neurological function test, cerebral blood flow detection, and cerebral infarction volume measurement immediately after they recovered from anesthesia while rats in the control group received all these measurement 60 hours later together with the acupuncture group. The experimental protocols were shown in Figure 1. A modified thread ligation method invented by Longa et al. [24] was applied to duplicate the model of middle cerebral artery occlusion. Briefly, rats were fasted for 12 hours with free access to water and anesthetized by intraperitoneal injection of $10 \%$ chloral hydrate $(350 \mathrm{mg} / \mathrm{kg})$. Rats were then fixed in the dorsal position on the surgery board, neck skin and muscle were incised, and the common carotid artery, the external carotid artery, and the internal carotid artery on the left were isolated. The external carotid artery and the proximal end (near the heart) of the common carotid were ligatured with 0 -suture line. A small hole was pierced with a $1 \mathrm{~mL}$ syringe needle at the proximal end of the common carotid. A $0.28 \mathrm{~mm}$ nylon thread was inserted from the hole into the internal carotid until resistance was met, with an intracranial depth of $18-20 \mathrm{~mm}$. Blood flow in the left middle cerebral artery was blocked by the nylon thread. The nylon thread was then ligatured with the common carotid artery and muscle and skin were sutured. The nylon thread was not applied in rats 
of sham group. This intraluminal suture model of MCAo produces reliable and permanent focal cerebral ischemia.

2.2. Acupuncture Intervention Methods. To rats in the acupuncture group, Neiguan (PC 6) acupoint on the right forelimb was needled with twisting-rotating method in the frequency of 3 times per second for 60 seconds. According to the Acupoint Location of Commonly Used Experimental Animals in the Experimental Acupuncture Science [25], Neiguan (PC 6) acupoint was located at the forelimb, between the ulna and the radius, about $3 \mathrm{~mm}$ from the wrist, perpendicularly needled $2 \mathrm{~mm}$ in depth. Sterile disposable stainless steel needles (length: $40 \mathrm{~mm}$, diameter: $0.30 \mathrm{~mm}$; Hwato, Suzhou Medical Supplies Factory Co., Ltd., China) were used in this study. All the acupuncture manipulation was operated by a skilled acupuncturist. Rats were needled for the first time right after they recovered from MCAo surgery and received 5 more times of acupuncture treatment in the subsequent 60 hours, 12 hours once.

To the sham and control groups, rats did not receive any acupuncture intervention but were also grabbed six times with the acupuncture group in the experimental period. To the model group, rats did not receive any acupuncture intervention. They received neurobehavioral test and cerebral blood flow (CBF) detection as soon as they recovered from anesthesia and then their brains were immediately collected for cerebral infarction volume measurement and microarray test.

2.3. Neurological Function Test. Neurological function test was performed according to Zausinger's six-point methods [26] once rats recovered from anesthesia. The standards used to obtain Zausinger's six-point score were as follows: score 0 , the rat could not spontaneously walk; score 1, the rat rotated towards the side opposite to the lesion with free walking; score 2 , the rat rotated towards the side opposite to the lesion when its tail was seized; score 3 , the resistance to the lateral pressure was decreased in the side opposite to the lesion; score 4 , the rat could not unbend front paws or entire forelimb on the side opposite to the lesion; score 5 , the rat had no neurological function defect. Only rats with a score of 1-3 were considered to be successful cerebral ischemia models and rats with a score of 0,4 , or 5 were not used for further experimentation. At 60 hours after modeling, all rats were assessed for another time except for the model group.

2.4. CBF Detection. CBF was measured by laser Doppler flowmetry (DRT4, Moor Instrument, Wilmington, DE, USA). Rats were fixed on a self-made stereotaxic instrument after anesthesia. A midline incision was made on the scalp to expose the anterior fontanel. A small bone window $1 \mathrm{~mm}$ posterior to the anterior fontanel and $3 \mathrm{~mm}$ left to the midline was produced with a dental drill. The measurement probe was placed on the left cerebral hemisphere to monitor moving red blood cells. CBF was continuously measured for 1 minute for each rat. Rats in the model group were immediately measured after they recovered from anesthesia. Rats in the sham and control groups were measured 60 hours later together with the acupuncture groups.
2.5. Brain Collection and Cerebral Infarction Volume Measurement. After neurological function test and CBF detection, six rats were randomly selected from each group for microarray test and others were conducted for cerebral infarction volume measurement. All rats were euthanized with chloral hydrate and their brains were collected quickly. As to the rats for microarray test, their left hemispheres were separated and kept at a temperature of $-80^{\circ} \mathrm{C}$ for mRNA extraction later. To other rats, their brains were placed at a temperature of $-20^{\circ} \mathrm{C}$ for 30 minutes and each then was cut into 5 coronal sections each $3 \mathrm{~mm}$ thick and stained with a $2 \%$ solution of 2,3,5-triphenyltetrazolium chloride (TTC) in phosphate buffered saline (PBS) at a temperature of $37^{\circ} \mathrm{C}$ for $20 \mathrm{~min}$, followed by $4 \%$ paraformaldehyde buffer for fixation. The stained sections were photographed by Olympus fe- 240 digital camera (Pooher Photoelectric Technology Co., Ltd., Shanghai, China) and the digital images were analyzed by a computer-assisted image system with Image-Pro Plus 6.0 (Rockville, MD, USA). The infarction volume was presented as a percentage of the total ipsilateral hemispheric volume which can be calculated by following equation: [(the volume of the intact contralateral hemisphere) - (the volume of the intact ipsilateral hemisphere - the volume of infarcted tissue in the ipsilateral hemisphere)]/(the volume of the intact contralateral hemisphere) $\times 100 \%$. This rectified measurement equation corrects for edema in the total infarct volume.

2.6. RNA Extraction. Total RNA was extracted from ipsilateral hemispheres of MCAo rats and the corresponding hemispheres of sham rats with TRIZOL Reagent (GibcoBRL, Rockville, MD) according to manufacturers' protocol and its quality was measured by NANODROP 2000 spectrophotometer (Thermo Scientific, USA) and 2100 Bioanalyzer (Agilent Technologies, Inc., USA). The total RNA was then purified with Qiagen RNeasy Mini Kit (Qiagen Inc., USA) and quantified by spectrophotometer and stored at $-80^{\circ} \mathrm{C}$ for microarray analysis and Real-Time Quantitative Polymerase Chain Reaction (RT-PCR) studies.

2.7. Microarray Analysis. An equal amount of total RNA $(100 \mathrm{ng})$ was used for the synthesis of cDNA and then cDNA was cleaned and used for cy3CTP, cy5CTP-labeled cRNA synthesis with T7 RNA polymerase. The labeled and fragmented cRNA was then hybridized to Agilent G4853A Gene-Chip (Agilent Technologies, Inc., USA) which contains 30,003 functional genes for 17 hours with required antisense controls. The microarray chips were then washed and scanned using Agilent Scan Control software (Agilent Technologies, Inc., USA) and data was analyzed by Agilent GeneSpring software GX10.05 (Agilent Technologies, Inc., USA). Differentially expressed genes were selected based on $P$ value $<0.05$ and $\log _{2}$ (Fold Change) $\geq 1$ or $\log _{2}$ (Fold Change) $\leq-1$.

2.8. KEGG Pathway Analyses for mRNAs. The Kyoto Encyclopedia of Genes and Genomes (KEGG) is a knowledge base for systematic analysis of gene functions, linking genomic information with higher order function information. KEGG pathway database is supplemented by a set of ortholog group 
TABLE 1: Primer names, sequences, and PCR conditions used for RT-PCR analysis.

\begin{tabular}{|c|c|c|c|c|}
\hline Gene & NCBI reference sequence & Primer sequence & $\operatorname{Tm}\left({ }^{\circ} \mathrm{C}\right)$ & Amplicon size (bp) \\
\hline \multirow{2}{*}{ Tph1 } & \multirow{2}{*}{ NM_001100634.2 } & $5^{\prime}$ GGCTTTGAGGTCCTCTTTCCA3 $3^{\prime}$ & \multirow{2}{*}{56} & \multirow{2}{*}{112} \\
\hline & & 5'CCCCСTTTCTGAGGAATGGTC3 ${ }^{\prime}$ & & \\
\hline \multirow{2}{*}{ Olr883 } & \multirow{2}{*}{ NM_001001358.1 } & 5'GCAGGCCACTGCACTATTTG3' & \multirow{2}{*}{56} & \multirow{2}{*}{123} \\
\hline & & 5'ACTGCAGATTTAGGCCGAGG3' & & \\
\hline \multirow{2}{*}{ Actin } & \multirow{2}{*}{ NM_031144.3 } & 5'CAGCCTTCCTTCCTGGGTATG3' & \multirow{2}{*}{55} & \multirow{2}{*}{247} \\
\hline & & $5^{\prime}$ TAGAGCCACCAATCCACACAG3' & & \\
\hline
\end{tabular}

tables for the information about conserved subpathways (pathway motifs), which are often encoded by positionally coupled genes on the chromosome and are especially useful in predicting gene functions [27]. Differently expressed genes were used for KEGG pathway analysis between groups (model versus sham, control versus acupuncture). Hypergeometric Distribution method was applied to calculate the $P$ value of each pathway and then rectified by false discovery rate (FDR) method. Possibility values of $<0.05$ were considered statistically significant.

2.9. Real-Time Quantitative Polymerase Chain Reaction Validation. Real-Time Quantitative Polymerase Chain Reaction (RT-PCR) was carried out for mRNA validation according to manufacturer's protocols using mRNA specific primers from Applied Biosystems (USA). Two genes, that is, Tph1 and Olr883, were chosen from all the differentially expressed genes. The complementary DNAs were synthesized by Shanghai Science \& Technical Co. (Shanghai, China). All samples were normalized by Rattus norvegicus actin. The information about primers used for RT-PCR was shown in Table 1. Each sample was tested in triplicate. Relative gene expression was measured by $2^{-\Delta \Delta \mathrm{CT}}$ method [28].

2.10. Statistical Analysis. All data in our study were analyzed by SPSS 16.0 software and presented as mean \pm SD except for microarray analysis. One-way analysis of variance (ANOVA) followed by the least significant difference (LSD) was used for analyzing the data. The standard statistical function of Hypergeometric Distribution, $t$-test, and FDR was performed for determined genes differently expressed in microarray analysis and KEGG pathways. Possibility values of $<0.05$ were considered statistically significant.

\section{Results}

3.1. Acupuncture Improved Neurological Function of MCAo Rats. Compared with the sham group, neurological deficit scores significantly decreased in the model group $(P<0.05)$ but improved after 60 hours in the acupuncture group $(P<$ 0.05). No significant difference was seen between the model and control groups $(P>0.05)$ while the acupuncture group had an apparent improvement compared with the control group $(P<0.05)$ (see Figure 2$)$.

3.2. Acupuncture Increased Cerebral Blood Flow (CBF) of $M C A o$ Rats. CBF was significantly reduced in the model control group compared with the sham group $(P<0.05)$

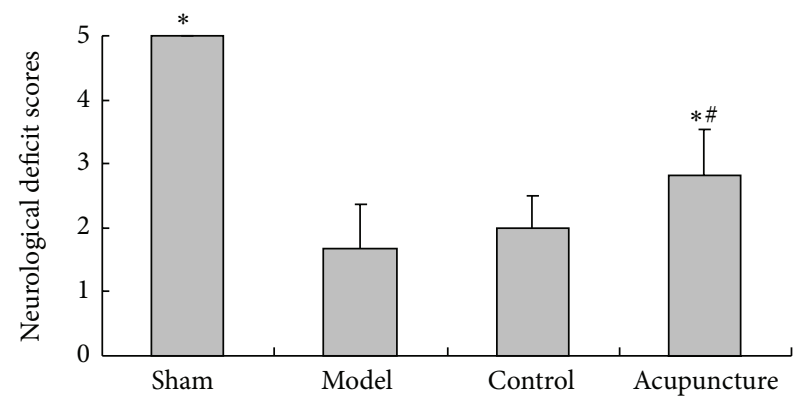

FIgURE 2: Effects of acupuncture on neurological deficit scores in MCAo rats: values are mean $\pm \mathrm{SD}(n=18)$ and ${ }^{*} P<0.05$ compared with the model group, ${ }^{\#} P<0.05$ compared with the control group.

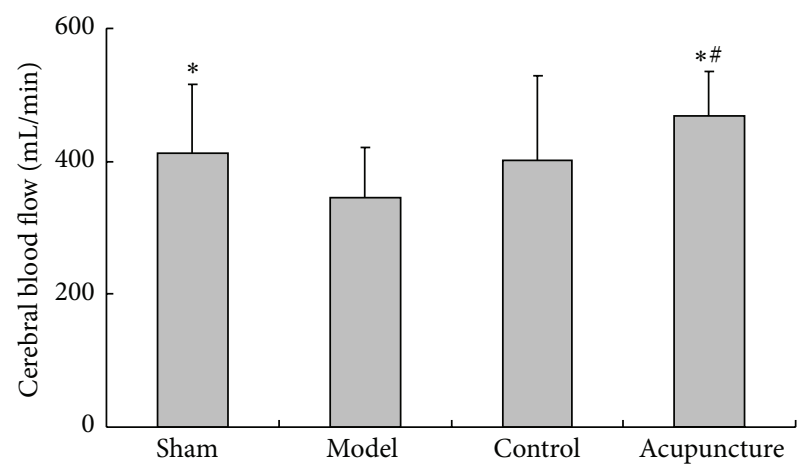

FIgURE 3: Effects of acupuncture on CBF in MCAo rats: values are mean $\pm \operatorname{SD}(n=18)$ and ${ }^{*} P<0.05$ compared with the model group, ${ }^{\#} P<0.05$ compared with the control group.

and increased after 60 hours in the acupuncture group $(P<$ 0.05). No significant difference was seen between the model and control groups $(P>0.05)$ while the acupuncture group had an apparent increase compared with the control group $(P<0.05)$ (see Figure 3).

3.3. Acupuncture Reduced Cerebral Infarction Volume of MCAo Rats. Compared with the sham group (zero), clear cerebral infarction volume was seen in the model group $(P<$ $0.05)$ and reduced in the acupuncture group $(P<0.05)$. No significant difference was seen between the model and control groups $(P>0.05)$ (see Figure 4$)$.

3.4. Changes in mRNA Expression and Pathways Involved in the Process of Ischemic Stroke. A total of 627 different 


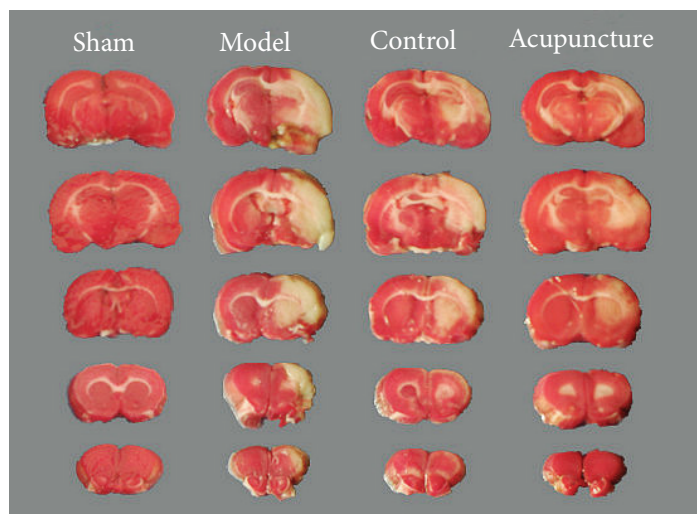

(a)

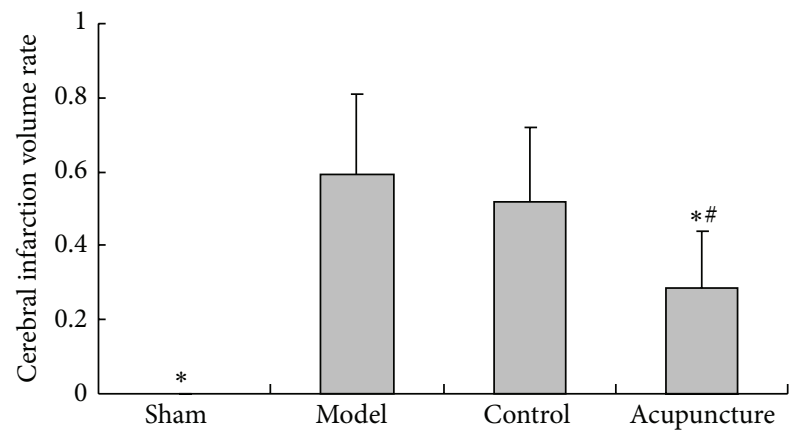

(b)

FIGURE 4: Effects of acupuncture on infarction volume. (a) Representative coronal sections of rat brains stained by TTC from four groups. The white area is infarct region and red is normal tissue. (b) Quantitative analysis of infarction volume. Values are mean \pm SD $(n=12)$ and ${ }^{*} P<0.05$ compared with the model group, ${ }^{\#} P<0.05$ compared with the control group.

TABLE 2: The top 10 upregulated genes of the model group versus the sham group.

\begin{tabular}{|c|c|c|c|c|}
\hline Gene symbol & Gene description & $\log _{2}(\mathrm{FC})$ & $P$ value & $P$.adjust \\
\hline Hspalb & Heat shock $70 \mathrm{kD}$ protein $1 \mathrm{~B}$ (mapped) & 6.91524 & $3.64 E-07$ & $3.96 E-04$ \\
\hline $\mathrm{Ccl} 2$ & Chemokine (C-C motif) ligand 2 & 6.85826 & $1.72 E-06$ & $1.16 E-03$ \\
\hline Cxcl2 & Chemokine (C-X-C motif) ligand 2 & 6.37562 & $7.87 E-05$ & $7.37 E-03$ \\
\hline Ccl3 & Chemokine (C-C motif) ligand 3 & 5.67107 & $7.19 E-06$ & $2.43 E-03$ \\
\hline Serpina3n & Serine (or cysteine) peptidase inhibitor, clade A, member $3 \mathrm{~N}$ & 5.61058 & $2.11 E-05$ & $4.16 E-03$ \\
\hline Timp1 & TIMP metallopeptidase inhibitor 1 & 5.60194 & $7.64 E-05$ & $7.34 E-03$ \\
\hline H19 & H19, imprinted maternally expressed transcript (nonprotein coding) & 5.43809 & $4.25 E-06$ & $1.80 E-03$ \\
\hline Hspb1 & Heat shock protein 1 & 5.41504 & $1.15 E-08$ & $5.62 E-05$ \\
\hline $\mathrm{Ccl} 20$ & Chemokine (C-C motif) ligand 20 & 5.20095 & $3.47 E-04$ & $1.51 E-02$ \\
\hline Cxcl1 & Chemokine (C-X-C motif) ligand 1 (melanoma growth stimulating activity, alpha) & 5.17590 & $4.94 E-06$ & $1.91 E-03$ \\
\hline
\end{tabular}

expression genes were regulated during the process of ischemic stroke. 417 genes were upregulated and 210 genes were downregulated.

Among the upregulated genes, $\mathrm{Hspalb}, \mathrm{Ccl} 2, \mathrm{Cxcl} 2, \mathrm{Ccl} 3$, Serpina3n, Timp1, H19, Hspb1, Ccl20, and Cxcl1 were found to demonstrate significant upregulation in pathological phase of ischemic stroke (see Table 2). 58 pathways were examined for upregulated mRNAs in this study including the regulation of Staphylococcus aureus infection, phagosome, cell adhesion molecules, cytokine-cytokine receptor interaction, hematopoietic cell lineage, Toll-like receptor signaling pathway, natural killer cell mediated cytotoxicity MAPK signaling pathway, p53 signaling pathway, VEGF signaling pathway, and TGF-beta signaling pathway (see Table 3 ).

Among the downregulated genes, Gpr88, Rgs9, Enthd1, Olr59, P2ry12, Degs2, Pde10a, Neu2, Adora2a, and Slc22a6 were found to demonstrate significant downregulation in pathological phase of ischemic stroke (see Table 4). 19 pathways of downregulated mRNAs were examined including the regulation of calcium signaling pathway, gastric acid secretion, salivary secretion, axon guidance, hypertrophic cardiomyopathy, neuroactive ligand-receptor interaction, longterm depression, axon guidance, pancreatic secretion, and sphingolipid metabolism (see Table 5).
3.5. Changes in mRNA Expression and Pathways Involved in Acupuncture Intervention. A total of 361 different expression genes were regulated during the process of acupuncture intervention. Three genes were upregulated and 358 genes were downregulated.

The three upregulated genes were Tph1, Loc684158, and Ccdc19 (see Table 6). No pathway was found among the 3 upregulated mRNAs, so pathway annotation was adopted and two pathways were involved, that is, tryptophan metabolism and metabolism (see Table 7).

Among the downregulated genes, Gpr88, Rgs9, Enthd1, Olr59, P2ry12, Degs2, Pde10a, Neu2, Adora2a, and Slc22a6 were found to demonstrate significant downregulation in pathological phase of ischemic stroke (see Table 8). The pathway of acupuncture intervention for downregulated mRNAs examined in this study is olfactory transduction (see Table 9).

3.6. Verification of Gene Expression By RT-PCR. RT-PCR results of Tph1 and Olr883 showed that directional changes of mRNA levels were in agreement with microarray analysis results. mRNA expression of $T p h 1$ was reduced in the model group and increased in the acupuncture group $(P<0.05)$. No difference was seen between the control and model groups $(P>0.05)$ (see Figure 5(a)). mRNA expression of Olr883 was 
TABLE 3: The enriched pathways of the up-regulated genes of the model group versus the sham group.

\begin{tabular}{|c|c|c|c|}
\hline KEGG ID & Description & $P$ value & Gene name \\
\hline 5144 & Malaria & $6.08 E-14$ & $\begin{array}{c}\text { Selp; Il6; Ccl12; Il1b; Cd36; Tnf; Tlr2; RGD1565355; Tgfb1; Itgb2; Thbs4; Icam1; } \\
\text { Cd40; Sele; Myd88; Sdc1 }\end{array}$ \\
\hline 5140 & Leishmaniasis & $1.15574 E-13$ & $\begin{array}{c}\text { Ncf4; Il1b; Jun; Fcgr3a; Tnf; Tlr2; Ptpn6; Fcgr1a; Fos; Ptgs2; Tgfb1; Itgam; Itgb2; } \\
\text { RT1-DMa; Nfkbia; Myd88; LOC498276 }\end{array}$ \\
\hline 5150 & $\begin{array}{l}\text { Staphylococcusaureus } \\
\text { infection }\end{array}$ & $1.70375 E-12$ & $\begin{array}{c}\text { Selp; Clqc; Fcgr3a; C1s; C3ar1; Fcgr1a; Itgam; Fcgr2b; Itgb2; RT1-DMa; C5ar1; } \\
\text { Icam1; LOC498276; Clqb }\end{array}$ \\
\hline 5323 & Rheumatoid arthritis & $5.37292 E-11$ & $\begin{array}{c}\text { Il6; Ccl12; Il1b; Jun; Tnf; Tlr2; Csf1; Cd86; Fos; Ccl20; Tgfb1; Itgb2; RT1-DMa; } \\
\text { Icam1; Ccl3; Ill1 }\end{array}$ \\
\hline 4380 & $\begin{array}{l}\text { Osteoclast } \\
\text { differentiation }\end{array}$ & $5.73512 E-11$ & $\begin{array}{c}\text { Ncf4; Il1b; Jun; Fcgr3a; Tyrobp; Tnf; Lcp2; Spil; Csf1; Fcgrla; Fos; Nfkb2; Tgfb1; } \\
\text { Fcgr2b; Fosl1; Nfkbia; Socs3; Tnfrsfla; LOC498276 }\end{array}$ \\
\hline 4145 & Phagosome & $1.7585 E-10$ & $\begin{array}{c}\text { Msr1; RT1-EC2; Ncf4; Fcgr3a; Cd36; RT1-A2; RT1-A1; Tap1; Tlr2; RGD1565355; } \\
\text { Fcgr1a; Tubb6; Itga5; Cd14; Itgam; Fcgr2b; Itgb2; RT1-CE5; RT1-DMa; Thbs4; } \\
\text { Olr1; RT1-CE2; LOC498276 }\end{array}$ \\
\hline 4514 & $\begin{array}{l}\text { Cell adhesion molecules } \\
\text { (CAMs) }\end{array}$ & $2.37222 E-10$ & $\begin{array}{c}\text { Ptprc; RT1-EC2; Selp; RT1-A2; RT1-A1; Cldn14; Cldn23; Cd86; Esam; Itgam; } \\
\text { PVR; Itgb2; RT1-CE5; RT1-DMa; Cd274; Icam1; Cd40; Sele; RT1-CE2; Sdc1; } \\
\text { Glycam1 }\end{array}$ \\
\hline 4060 & $\begin{array}{l}\text { Cytokine-cytokine } \\
\text { receptor interaction }\end{array}$ & $1.65905 E-09$ & $\begin{array}{l}\text { Il6; Ccl12; Il1b; Cxcl16; Tnf; Ccl4; Clcf1; Csf1; Cxcl2; Illr2; Cxcl10; Tnfrsf12a; } \\
\text { Tnfrsf1b; Ccl20; Il4ra; Tgfb1; Ltbr; Il13ra1; Cd40; Tnfrsfla; Ccl3; Il11; Csf2rb }\end{array}$ \\
\hline 4640 & $\begin{array}{l}\text { Hematopoietic cell } \\
\text { lineage }\end{array}$ & $2.81713 E-09$ & $\begin{array}{c}\text { Il6; Il1b; Cd36; Tnf; Csf1; RGD1565355; Fcgrla; Itga5; Illr2; Cd14; Il4ra; Itgam; } \\
\text { Cd44; Il11 }\end{array}$ \\
\hline 4620 & $\begin{array}{l}\text { Toll-like receptor } \\
\text { signaling pathway }\end{array}$ & $3.71149 E-09$ & $\begin{array}{l}\text { Il6; Il1b; Jun; Tnf; Tlr2; Cd86; Cxcl10; Cd14; Fos; Lbp; Cd40; Nfkbia; Ccl3; } \\
\text { Myd88; Spp1 }\end{array}$ \\
\hline 4610 & $\begin{array}{l}\text { Complement and } \\
\text { coagulation cascades }\end{array}$ & $3.91282 E-09$ & $\begin{array}{l}\text { Serpine1; C1qc; Fga; Kng1; Plaur; C1s; C3arl; Serping1; A2m; C6; C5ar1; F10; } \\
\text { C1qb }\end{array}$ \\
\hline 5142 & $\begin{array}{l}\text { Chagas disease } \\
\text { (American } \\
\text { trypanosomiasis) }\end{array}$ & $1.28382 E-08$ & $\begin{array}{c}\text { Serpine1; C1qc; Il6; Ccl12; Il1b; Jun; Tnf; Tlr2; Fos; Tgfb1; Nfkbia; Tnfrsfla; Ccl3; } \\
\text { Myd88; Clqb }\end{array}$ \\
\hline 4062 & $\begin{array}{l}\text { Chemokine signaling } \\
\text { pathway }\end{array}$ & $7.78649 E-08$ & $\begin{array}{c}\text { Ccl2; Hck; Ccl6; Ccl7; Ccl12; Shcl; Cxcl16; Cxcl1; Ccl4; Cxcl2; Adcy4; Cxcl10; Fgr; } \\
\text { Rac2; Ccl20; Stat3; Nfkbia; Ccl3; Jak3 }\end{array}$ \\
\hline 5332 & $\begin{array}{l}\text { Graft-versus-host } \\
\text { disease }\end{array}$ & $5.76242 E-07$ & RT1-EC2; Il6; Il1b; RT1-A2; RT1-A1; Tnf; Cd86; RT1-CE5; RT1-DMa; RT1-CE2 \\
\hline 4612 & $\begin{array}{l}\text { Antigen processing and } \\
\text { presentation }\end{array}$ & $1.07678 E-06$ & $\begin{array}{c}\text { RT1-EC2; RT1-A2; RT1-A1; Tap1; Tnf; Ifi30; Hspa2; Lgmn; Hspa1b; RT1-CE5; } \\
\text { RT1-DMa; RT1-CE2 }\end{array}$ \\
\hline 4010 & $\begin{array}{l}\text { MAPK signaling } \\
\text { pathway }\end{array}$ & $1.18683 E-06$ & $\begin{array}{l}\text { Gadd45g; Myc; Il1b; Hspb1; Jun; Flna; Tnf; Flnc; Gadd45b; Fgf2; Hspa2; Illr2; } \\
\text { Cd14; Rac2; Fos; Map3k6; Nfkb2; Tgfb1; Gadd45a; Hspa1b; Tnfrsfla; Dusp5 }\end{array}$ \\
\hline 4621 & $\begin{array}{l}\text { NOD-like receptor } \\
\text { signaling pathway }\end{array}$ & $1.44958 E-06$ & Il6; Ccl12; Il1b; Cxcl1; Tnf; Birc3; Cxcl2; Pycard; Nfkbia \\
\hline 5146 & Amoebiasis & $2.86186 E-06$ & Il6; Il1b; Hspb1; Tnf; Tlr2; Col4a1; Il1r2; Cd14; Col4a2; Tgfb1; Itgam; Itgb2 \\
\hline 4512 & $\begin{array}{l}\text { ECM-receptor } \\
\text { interaction }\end{array}$ & $5.01276 E-06$ & Cd36; Tnc; RGD1565355; Col4a1; Itga5; Col4a2; Thbs4; Cd44; Sdc1; Spp1 \\
\hline 5330 & Allograft rejection & $6.43761 E-06$ & RT1-EC2; RT1-A2; RT1-A1; Tnf; Cd86; RT1-CE5; RT1-DMa; Cd40; RT1-CE2 \\
\hline 4920 & $\begin{array}{l}\text { Adipocytokine signaling } \\
\text { pathway }\end{array}$ & $1.72078 E-05$ & Cd36; Tnf; RGD1565355; Tnfrsfib; Prkab2; Stat3; Nfkbia; Socs3; Tnfrsf1a \\
\hline 4940 & Type I diabetes mellitus & $1.72078 E-05$ & RT1-EC2; Il1b; RT1-A2; RT1-A1; Tnf; Cd86; RT1-CE5; RT1-DMa; RT1-CE2 \\
\hline 5416 & Viral myocarditis & $1.7893 E-05$ & $\begin{array}{c}\text { RT1-EC2; RT1-A2; RT1-A1; Cd86; Rac2; Itgb2; RT1-CE5; RT1-DMa; Icam1; } \\
\text { Cd40; RT1-CE2 }\end{array}$ \\
\hline 4623 & $\begin{array}{l}\text { Cytosolic DNA-sensing } \\
\text { pathway }\end{array}$ & $4.3625 E-05$ & Il6; Illb; Ripk3; Ccl4; Cxcl10; Pycard; Nfkbia \\
\hline 5143 & African trypanosomiasis & $4.73199 E-05$ & Il6; Il1b; Tnf; Icam1; Sele; Myd88 \\
\hline 5145 & Toxoplasmosis & $5.71133 E-05$ & $\begin{array}{c}\text { Tnf; Tlr2; Birc3; Hspa2; Tgfb1; Stat3; Hspa1b; RT1-DMa; Cd40; Nfkbia; Tnfrsf1a; } \\
\text { Myd88 }\end{array}$ \\
\hline 5322 & $\begin{array}{l}\text { Systemic lupus } \\
\text { erythematosus }\end{array}$ & $7.24914 E-05$ & $\begin{array}{c}\text { Clqc; Fcgr3a; Tnf; C1s; Cd86; Fcgrla; Fcgr2b; RT1-DMa; C6; Cd40; LOC498276; } \\
\text { Clqb }\end{array}$ \\
\hline
\end{tabular}


TABLE 3: Continued.

\begin{tabular}{|c|c|c|c|}
\hline KEGG ID & Description & $P$ value & Gene name \\
\hline 4670 & $\begin{array}{c}\text { Leukocyte } \\
\text { transendothelial } \\
\text { migration }\end{array}$ & $7.3107 E-05$ & $\begin{array}{c}\text { Ncf4; Mmp9; Cldn14; Msn; Cldn23; Esam; RGD1309537; Rac2; Itgam; Itgb2; } \\
\text { Icam1 }\end{array}$ \\
\hline 4650 & $\begin{array}{l}\text { Natural killer cell } \\
\text { mediated cytotoxicity }\end{array}$ & 0.000108813 & Fcgr3a; Shc1; Tyrobp; Tnf; Lcp2; Ptpn6; Rac2; Itgb2; Icam1; Fcerlg \\
\hline 5320 & $\begin{array}{l}\text { Autoimmune thyroid } \\
\text { disease }\end{array}$ & 0.000149638 & RT1-EC2; RT1-A2; RT1-A1; Cd86; RT1-CE5; RT1-DMa; Cd40; RT1-CE2 \\
\hline 4672 & $\begin{array}{c}\text { Intestinal immune } \\
\text { network for IgA } \\
\text { production }\end{array}$ & 0.000155052 & Il6; Cd86; Tgfb1; RT1-DMa; Ltbr; Cd40 \\
\hline 5310 & Asthma & 0.000428565 & Tnf; RT1-DMa; Cd40; Fcerlg \\
\hline 5020 & Parkinson's disease & 0.000474519 & C1qc; Il6; Il1b; C6; C1qb \\
\hline 4510 & Focal adhesion & 0.000611017 & $\begin{array}{c}\text { Jun; Flna; Shc1; Tnc; Birc3; Col4a1; Flnc; Itga5; RGD1309537; Rac2; Col4a2; } \\
\text { Thbs4; Spp1 }\end{array}$ \\
\hline 4630 & $\begin{array}{l}\text { Jak-STAT signaling } \\
\text { pathway }\end{array}$ & 0.000690892 & Myc; Il6; Ptpn6; Clcf1; Il4ra; Stat3; Il13ra1; Socs3; Il11; Csf2rb; Jak3 \\
\hline 4666 & $\begin{array}{l}\text { Fc gamma R-mediated } \\
\text { phagocytosis }\end{array}$ & 0.001026538 & Ptprc; Hck; Fcgrla; Arpc1b; Sphk1; Rac2; Fcgr2b; LOC498276 \\
\hline 4115 & p53 signaling pathway & 0.001031142 & Gadd45g; Serpine1; Igfbp3; Gadd45b; RGD1566319; Gadd45a; LOC298795 \\
\hline 5200 & Pathways in cancer & 0.001310312 & $\begin{array}{c}\text { Ret; Myc; Il6; Jun; Mmp9; Runx1; Spil; Birc3; Col4a1; Fgf2; Rac2; Fos; Col4a2; } \\
\text { Ptgs2; Nfkb2; Tgfb1; Stat3; Nfkbia }\end{array}$ \\
\hline 5410 & $\begin{array}{c}\text { Hypertrophic } \\
\text { cardiomyopathy (HCM) }\end{array}$ & 0.001575221 & Il6; Tnf; Tpm4; Itga5; Des; Prkab2; Tgfb1 \\
\hline 5340 & $\begin{array}{c}\text { Primary } \\
\text { immunodeficiency }\end{array}$ & 0.002241893 & Ptprc; Tap1; Cd40; Jak3 \\
\hline 5221 & Acute myeloid leukemia & 0.003919315 & Myc; Runx1; Eif4ebp1; Spil; Stat3 \\
\hline 4210 & Apoptosis & 0.004080222 & Il1b; Tnf; Birc3; Nfkbia; Tnfrsfla; Myd88; Csf2rb \\
\hline 4662 & $\begin{array}{l}\text { B cell receptor signaling } \\
\text { pathway }\end{array}$ & 0.004536991 & Jun; Ptpn6; Rac2; Fos; Fcgr2b; Nfkbia \\
\hline 4110 & Cell cycle & 0.007661902 & Gadd45g; Myc; Mcm3; Gadd45b; Tgfb1; Gadd45a; LOC298795; Mcm6 \\
\hline 4622 & $\begin{array}{l}\text { RIG-I-like receptor } \\
\text { signaling pathway }\end{array}$ & 0.008260836 & Rnf125; Tnf; Cxcl10; Trim25; Nfkbia \\
\hline 5222 & Small cell lung cancer & 0.008411479 & Myc; Birc3; Col4a1; Col4a2; Ptgs2; Nfkbia \\
\hline 5160 & Hepatitis C & 0.00890146 & Irf1; Cldn14; Tnf; Cldn23; Stat3; Nfkbia; Socs3; Tnfrsfla \\
\hline 4660 & $\begin{array}{l}\text { T cell receptor signaling } \\
\text { pathway }\end{array}$ & 0.010477739 & Ptprc; Jun; Tnf; Lcp2; Ptpn6; Fos; Nfkbia \\
\hline 5414 & Dilated cardiomyopathy & 0.010752535 & Tnf; Tpm4; Adcy4; Itga5; Des; Tgfb1 \\
\hline 5210 & Colorectal cancer & 0.011794184 & Myc; Jun; Rac2; Fos; Tgfb1 \\
\hline 5220 & $\begin{array}{l}\text { Chronic myeloid } \\
\text { leukemia }\end{array}$ & 0.015301029 & Myc; Shcl; Runxl; Tgfbl; Nfkbia \\
\hline 4370 & VEGF signaling pathway & 0.017300772 & Nos3; Hspb1; Sphk1; Rac2; Ptgs2 \\
\hline 4144 & Endocytosis & 0.025647683 & $\begin{array}{c}\text { RT1-EC2; Ret; Ehd2; RT1-A2; RT1-A1; Hspa2; Dab2; Tgfb1; Hspalb; RT1-CE5; } \\
\text { RT1-CE2 }\end{array}$ \\
\hline 4350 & $\begin{array}{l}\text { TGF-beta signaling } \\
\text { pathway }\end{array}$ & 0.030013655 & Myc; Tnf; Bmp7; Tgfb1; Thbs4 \\
\hline 4012 & ErbB signaling pathway & 0.030013655 & Myc; Jun; Shc1; Eif4ebpl; Hbegf \\
\hline 4141 & $\begin{array}{l}\text { Protein processing in } \\
\text { endoplasmic reticulum }\end{array}$ & 0.038063498 & Hspa2; Dnaja1; Hspa1b; Cryaa; Cryab; Hsph1; Sec24a; Ppp1r15a \\
\hline 4930 & Type II diabetes mellitus & 0.045976221 & Tnf; Hk2; Socs3 \\
\hline 5100 & $\begin{array}{l}\text { Bacterial invasion of } \\
\text { epithelial cells }\end{array}$ & 0.046356305 & Shc1; Arpc1b; Itga5; Hcls1 \\
\hline
\end{tabular}


TABLE 4: The top 10 downregulated genes of the model group versus the sham group.

\begin{tabular}{|c|c|c|c|c|}
\hline Gene symbol & Gene description & $\log _{2}(\mathrm{FC})$ & $P$ value & $P$.adjust \\
\hline Gpr88 & G-protein coupled receptor 88 & -2.24907 & 0.00163 & 0.02984 \\
\hline $\operatorname{Rgs} 9$ & Regulator of G-protein signaling 9 & -2.17074 & 0.00035 & 0.01507 \\
\hline Enthd1 & ENTH domain containing 1 & -1.95721 & 0.00005 & 0.00606 \\
\hline Olr59 & Olfactory receptor 59 & -1.90729 & 0.00238 & 0.03558 \\
\hline P2ry12 & Purinergic receptor P2Y, G-protein coupled, 12 & -1.85441 & 0.00116 & 0.02520 \\
\hline Degs2 & $\operatorname{Delta}(4)$-desaturase, sphingolipid 2 & -1.85094 & 0.00198 & 0.03247 \\
\hline Pde10a & Phosphodiesterase 10A & -1.78373 & 0.00000 & 0.00012 \\
\hline $\mathrm{Neu} 2$ & Sialidase 2 (cytosolic sialidase) & -1.78245 & 0.00170 & 0.03040 \\
\hline Adora $2 a$ & Adenosine A2a receptor & -1.76237 & 0.00003 & 0.00478 \\
\hline Slc22a6 & Solute carrier family 22 (organic anion transporter), member 6 & -1.74866 & 0.00002 & 0.00416 \\
\hline
\end{tabular}

TABLE 5: The enriched pathways of the downregulated genes of the model group versus the sham group.

\begin{tabular}{|c|c|c|c|}
\hline KEGG ID & Description & $P$ value & Gene name \\
\hline 4260 & Cardiac muscle contraction & $1.19216 E-07$ & $\begin{array}{c}\text { Tnnt2; Myh7; Myh6; Atpla1; Atpla2; Tnni3; } \\
\text { Cox8b; Cacng3 }\end{array}$ \\
\hline 4020 & Calcium signaling pathway & $2.71115 E-07$ & $\begin{array}{l}\text { Plcb1; Cckbr; Drd1a; Tacr3; Slc8a2; Camk4; } \\
\quad \text { Adrald; Atp2b2; Itpr1; Pde1b; Adora2a }\end{array}$ \\
\hline 4971 & Gastric acid secretion & $8.46439 E-06$ & Plcb1; Cckbr; Kcnk2; Atp1a1; Atpla2; Itpr1 \\
\hline 4970 & Salivary secretion & $1.20354 E-05$ & Plcb1; Adrald; Atp2b2; Atp1a1; Atp1a2; Itpr1 \\
\hline 5410 & Hypertrophic cardiomyopathy (HCM) & $1.42423 E-05$ & Tnnt2; Itgb6; Myh7; Myh6; Tnni3; Cacng3 \\
\hline 4080 & Neuroactive ligand-receptor interaction & $2.28164 E-05$ & $\begin{array}{l}\text { Cckbr; Drd2; Drd1a; Grm3; Tacr3; Adrald; } \\
\text { Chrm4; Gria3; Htrld; Adora2a; Adora3 }\end{array}$ \\
\hline 5414 & Dilated cardiomyopathy & $2.66995 E-05$ & Tnnt2; Itgb6; Myh7; Myh6; Tnni3; Cacng3 \\
\hline 4360 & Axon guidance & 0.000275226 & Cxcl12; Ephb3; Robo2; Ngef; Lrrc4c; Epha4 \\
\hline 4972 & Pancreatic secretion & 0.000605555 & Plcb1; Atp2b2; Atp1a1; Atp1a2; Itpr1 \\
\hline 600 & Sphingolipid metabolism & 0.000811033 & Smpd3; Degs2; Neu2 \\
\hline 4976 & Bile secretion & 0.000867651 & Slc22a8; Slco1a2; Atp1a1; Atp1a2 \\
\hline 4974 & Protein digestion and absorption & 0.001102164 & Slc8a2; Col11a2; Atp1a1; Atp1a2 \\
\hline 4270 & Vascular smooth muscle contraction & 0.001284378 & Plcb1; Ramp1; Adra1d; Itpr1; Adora2a \\
\hline 4540 & Gap junction & 0.001989051 & Plcb1; Drd2; Drd1a; Itpr1 \\
\hline 4720 & Long-term potentiation & 0.005104314 & Plcb1; Camk4; Itpr1 \\
\hline 4730 & Long-term depression & 0.005650817 & Plcbl; Itpr1; Gria3 \\
\hline 4070 & Phosphatidylinositol signaling system & 0.007185071 & Plcbl; Dgkb; Itpr1 \\
\hline 4062 & Chemokine signaling pathway & 0.009027556 & Cxcl12; Plcb1; Rasgrp2; Gng7; Cx3cl1 \\
\hline 4530 & Tight junction & 0.045528155 & Cldn10; Myh7; Myh6 \\
\hline
\end{tabular}

increased in the model group and reduced in the acupuncture group $(P<0.05)$. No difference was seen between the control and model groups $(P>0.05)$ (see Figure 5(b)).

\section{Discussion}

To better understand the mechanisms of the therapeutic effect of acupuncture on ischemic stroke, we used microarray analysis in rat brain to examine the genomic response to ischemic stroke and acupuncture intervention. To the best of our knowledge, this is the first report using an mRNA microarray to survey global differential gene expression after manual acupuncture treatment in ischemic stroke animal models.
The microarray analysis performed during the period of ischemic stroke confirmed the bidirectional expressions of many genes. The gene expression of ischaemic stroke is characterised by differential expressions of a great number of genes involved in inflammation, apoptosis, protein turnover, transcription, signal transduction, ion-channel regulation, and metabolism which suggests that ischemic stroke is a multiple pathological process and it can trigger a multifaceted cascade of physiologic and biochemical events. These events are mediated in part by alterations of molecular transcriptional and translational activities. Our microarray findings are in accordance with previous studies of individual genes and pathways proposed to be associated with ischaemic brain injury $[29,30]$. Most of the differential genes were 
TABLE 6: The upregulated genes of the acupuncture group versus the control group.

\begin{tabular}{lcccc}
\hline Gene symbol & Gene description & $\log _{2}($ FC) & $P$ value & $P$. adjust \\
\hline Tph1 & Tryptophan hydroxylase 1 & 3.71189 & 0.04627 & 0.43944 \\
Loc684158 & Similar to chromosome 1 open reading frame 36 & 1.44377 & 0.00892 & 0.43944 \\
Ccdc19 & Coiled-coil domain containing 19 & 1.01508 & 0.03612 & 0.43944 \\
\hline
\end{tabular}

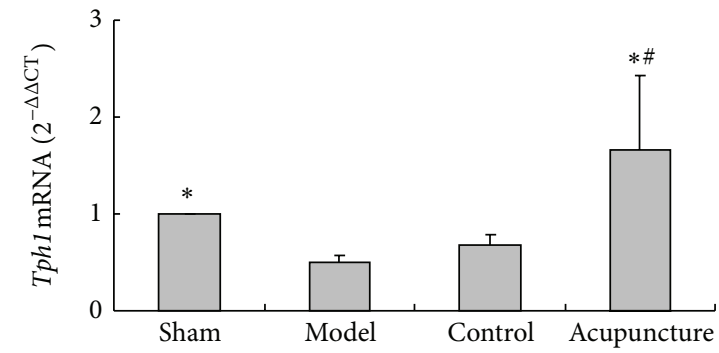

(a)

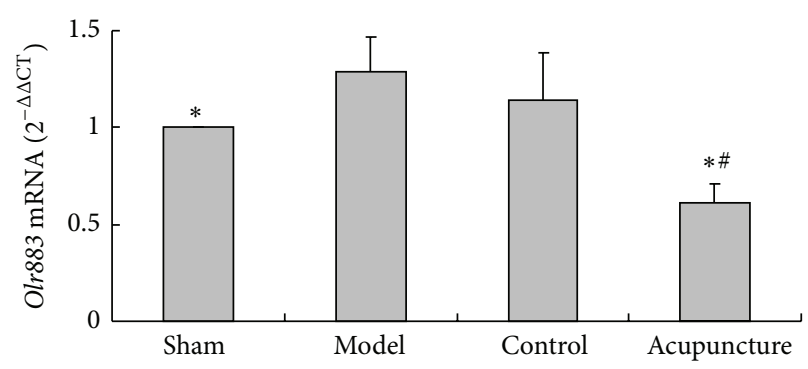

(b)

FIgURE 5: Analysis of mRNA expression of Tph1 and Olr883 by RT-PCR. (a) mRNA expression of Tph1 in the sham, model, control, and acupuncture groups. (b) mRNA expression of Olr883 in the sham, model, control, and acupuncture groups. Values are mean \pm SD $(n=6)$ and ${ }^{*} P<0.05$ compared with the model group, ${ }^{\#} P<0.05$ compared with the control group.

TABLe 7: Pathway annotation of the upregulated genes of the acupuncture group versus the control group.

\begin{tabular}{lccc}
\hline Gene ID & Gene name & KEGG ID & $\begin{array}{c}\text { KEGG } \\
\text { pathways }\end{array}$ \\
\hline 24848 & Tph1 & 380 & $\begin{array}{c}\text { Tryptophan } \\
\text { metabolism } \\
24848\end{array}$ \\
\hline
\end{tabular}

upregulated including heat shock protein (Hsp) genes and chemokine ligand genes. The upregulation of Hsp 70 genes such as Hspalb and Hspal after ischemic stroke in the present study is consistent with other studies $[29,31]$. As we all know, apoptosis-related genes are induced after focal ischemia, and they may contribute to cell death in the core and the selective cell death adjacent to an infarct [32], whereas heat shock proteins have been shown to play an important protective role in vivo as they have the ability to protect cultured cells from apoptosis and thus to protect brain against ischaemia [33]. Similar to heat shock proteins, an increase in mRNA expression of chemokines ( $\mathrm{Cl} 2, \mathrm{Ccl} 3, \mathrm{Cxcl} 2$, and $\mathrm{Ccl} 20$ ) was observed in the present study and this result is also consistent with other studies [31, 34]. Members of the CC and CXC chemokines are induced in the rat brain after focal cerebral ischemia and they are believed to contribute to secondary neuronal damage [29] through modulating crucial processes such as inflammatory cell recruitment and activation, neuronal survival, and neoangiogenesis. On the other hand, CXC ( $\mathrm{Cxcl} 2, \mathrm{Cxcl3})$ chemokines could also modulate stem cell homing, thus favouring tissue repair [35]. Therefore chemokine receptors were potential targets for therapeutic intervention in stroke [36]. Upregulated chemokines in the ischemic brain may reduce inflammatory cell migration to the brain in early stroke. Inhibition of inflammatory cell accumulation in the brain at the early stage of stroke may lead to amelioration of ischemic neurodegeneration $[37,38]$.

Compared with upregulated genes of ischemic stroke, information on downregulated genes seems relatively limited. Among the genes that were downregulated, G-protein genes were found to be closely related to ischemic injury. The signal transduction system mediated by G-protein is the most common way of signaling in cells. According to our microarray results, G-protein genes such as Gpr88 and $\operatorname{Rgs} 9$ were downregulated. Gpr88 may play a role in the fundamental functions of striatum such as the control of motor behavior [39]. A recent study reported that, in Gpr88 knockout mice, basal extracellular dopamine levels in the striatum were lower, while amphetamine-induced dopamine release was normal, and these mice also displayed increased apomorphineinduced stereotypy and amphetamine-stimulated locomotor activity [40]. These results suggest that Gpr88 plays a role in the regulation of dopamine signaling in the striatum and it may be a new target for treatments for psychiatric disorders after ischemic stroke. The $R g s 9$ gene gives rise to two splice forms $R g s 9-1$ and $R g s 9-2$. $R g s 9-1$ is expressed exclusively in retina, while Rgs9-2 is highly enriched in striatal regions of the brain. $\operatorname{Rgs} 9$ plays a role in striatal dopaminemediated behavior [41] and has been shown to be a critical negative regulator of opiate action in vivo [42]. Rgs 9 knockout rats exhibit normal locomotor activity, anxiety-like behavior, cued and contextual fear conditioning, startle threshold, and prepulse inhibition which suggest that $\operatorname{Rgs} 9-2$ is a potential therapeutic target for disorders involving motor or cognitive dysfunction [43].

Among all the different expression genes in the process of ischemic stroke, some may aggravate the obstruction of blood flow, death of ischemic neurons, and dysfunction of subsequent neurological behavior that had led to the early and ultimate pathological injury of ischemic stroke and some 
TABLE 8: The top 10 downregulated genes of the acupuncture group versus the control group.

\begin{tabular}{|c|c|c|c|c|}
\hline Gene symbol & Gene description & $\log _{2}(\mathrm{FC})$ & $P$ value & P.adjust \\
\hline Slpil3 & Antileukoproteinase-like 3 & -7.31439 & 0.01533 & 0.43944 \\
\hline Cyp2c6v1 & Cytochrome P450, family 2, subfamily C, polypeptide 6 , variant 1 & -7.22349 & 0.02509 & 0.43944 \\
\hline Dmrt2 & Doublesex and mab-3 related transcription factor 2 & -7.00699 & 0.03210 & 0.43944 \\
\hline Rgd1565502 & $\operatorname{Rgd1565502}$ & -7.00010 & 0.02472 & 0.43944 \\
\hline Olr1376 & Olfactory receptor 1376 & -6.87525 & 0.02725 & 0.43944 \\
\hline Loc680428 & Hypothetical protein $\operatorname{Loc} 680428$ & -6.84698 & 0.02102 & 0.43944 \\
\hline $\operatorname{Rgd1559709}$ & Similar to F-box protein 47 & -6.59986 & 0.03060 & 0.43944 \\
\hline Olr883 & Olfactory receptor 883 & -6.50528 & 0.03626 & 0.43944 \\
\hline Loc683282 & Similar to developmental pluripotency-associated 2 & -6.47640 & 0.03546 & 0.43944 \\
\hline Olr469 & Olfactory receptor 469 & -6.44318 & 0.02394 & 0.43944 \\
\hline
\end{tabular}

TABLE 9: The enriched pathways of the downregulated genes of the acupuncture group versus the control group.

\begin{tabular}{|c|c|c|c|}
\hline KEGG ID & Description & $P$ value & Gene name \\
\hline 4740 & Olfactory transduction & 0 & $\begin{array}{l}\text { Olr851; Olr1029; Olr883; Olr1507; Olr377; Olr1641; Olr259; Olr1652; Olr722; Olr690; } \\
\text { Olr1455; Olr1670; Olr807; Olr1616; Olr419; Olr1384; Olr1240; Olr1204; Olr424; Olr415; } \\
\text { Olr404; Olr1535; Olr606; Olr1146; Olr1643; Olr447; Olr469; Olr502; Olr1394; Olr1529; } \\
\text { Olr1530; Olr135; Olr344; Olr731; Olr639; Olr1314; Olr1311; Olr610; Olr607; Olr1326; } \\
\text { Olr575; Olr1365; Olr1376; Olr1549; Olr1588; Olr1600; Olr20; Olr85; Olr80; Olr47; } \\
\text { Olr219; Olr171; Olr170; Olr180; Olr121; Olr886; Olr1482; Olr1749; Olr1714; Olr533; } \\
\quad \text { Olr695; Olr737; Olr1060; Olr1389; Olr750; Olr758; Olr792 }\end{array}$ \\
\hline
\end{tabular}

may play an important protective role as the spontaneous anti-injury response in the pathological process of ischemic stroke. The microarray results also showed that ischemic stroke had a widespread influence on the whole body rather than a specific change in the brain; therefore therapeutic strategy aiming at the remedy of single pathological change might not obtain optimistic result.

According to previous researches, spontaneous recovery plays an important role in the pathological process of ischemic stroke [44]. In the present study, we observed the spontaneous recovery in 60 hours after modeling in MCAo rats; however, results did not show any sound outcomes. On the other hand, acupuncture intervention could effectively improve the neurological function and $\mathrm{CBF}$ and reduce the infarction volume of MCAo rats (see Figures 2, 3, and 4). Different patterns of gene expression occurred in the control and acupuncture groups. Our microarray analysis showed that most gene expression levels were downregulated following acupuncture treatment which suggests that the therapeutic effect of acupuncture on ischemic stroke may mainly depend on strategies to suppress the pathological progress of ischemic stroke instead of facilitating the repair of ischemic stroke injury. KEGG pathway analysis showed that the only pathway of the downregulated genes was olfactory transduction. Olfactory ensheathing cells (OECs) express adhesion molecules on their plasma membranes to help neural cells attach, and they also secrete a variety of trophic factors to facilitate neural regeneration and axon outgrowth. Intracerebral transplantation of OECs/olfactory nerve fibroblasts (ONFs), which secrete trophic factors including stromal cell-derived factor-la (SDF-1a), effectively leads to the recovery of the damaged cerebral tissue in murine models of stroke, thereby promoting the reversal of neurological deficit [45]. Transplantation of OECs has been shown to protect the white matter from ischemic injury [46], leading to improving neurological function in rats. Although there is relatively limited research of olfactory transduction in ischemia stroke, our result suggests that olfactory transduction might play an important role in ischemic stroke recovery and the therapeutic effect of acupuncture on ischemic stroke is closely related to the regulation of olfactory transduction.

Three genes were found upregulated after acupuncture intervention including Tph1, Loc684158, and Ccdc19. Pathway annotation showed tryptophan metabolism was concerned. Tph1 is a subtype of tryptophan hydroxylase (TPH) and TPH plays an important role in the biosynthesis of 5hydroxytryptamine (5-HT). Cerebral ischemia disturbs the normal metabolism of amine and decreases the bioavailability of amine in brain tissues which causes the hyposecretion of 5-hydroxytryptamine (5-HT), norepinephrine (NE), dopamine, and other neurotransmitters [47]. This kind of amine metabolism closely related to the mechanism of poststroke depression. At least $1 / 3$ stroke survivors have varying degrees of emotional symptoms [48, 49]. Depression is the most common symptom which increases the mortality, recurrence rate, and mobility of stroke patients [50-53]. TPH starts the synthesis process by catalyzing L-tryptophan into 5-HT and also acts as the rate limiting enzyme in the whole process. Therefore the activity of TPH directly influences the level and function of 5-HT. Our result of microarray analysis and RT-PCR result showed that the expression of Tphl was increased after acupuncture treatment which indicated that acupuncture had a potential effect on poststroke depression by promoting the level of 5-HT in brain. 
In conclusion, the present study discovered several characteristics of cerebral ischemia-induced gene expression and they may enrich our understanding of the multiple pathological process of ischemic brain injury. Meanwhile we also discovered therapeutic candidates stimulated or inhibited by acupuncture in ischemic stroke, and two genes were especially investigated. Upregulation of Tph1 and downregulation of Olr883 after acupuncture treatment indicate that the therapeutic effect of acupuncture for ischemic stroke may be closely related to the suppression of poststroke depression and regulation of olfactory transduction. These results may be useful in pursuing further studies on the possible mechanisms of acupuncture on ischemic stroke. Considering the difference between rats and human beings [54], subsequent clinical researches are also needed to further approve the findings of the current study.

\section{Conflict of Interests}

No conflict of interests is reported.

\section{Authors' Contribution}

Zhihong Meng and Xiaonong Fan conceived the study and prepared the initial protocol. Chao Zhang and Yan Wen drafted the paper and participated in the study design. Xueyi Zhou and Sha Yang participated in completing the study. Guang Tian and Yaqiong Chen helped to analyze the data. All authors approved the final version of the paper. Chao Zhang and Yan Wen contribute to the paper equally.

\section{Acknowledgments}

The authors sincerely thank all staff from Acupuncture and Moxibustion Research Institute of Tianjin University of Traditional Chinese Medicine for offering the experimental areas and instruments. Many thanks are given to all the research fellows in the present research for providing valuable guidance and selfless help. This study was supported by the National Basic Research Programs of China (973 Program), no. 2010CB530506.

\section{References}

[1] WHO, Global burden of Stroke, 2008, http://www.who.int/ cardiovascular_diseases/en/cvd_atlas_15_burden_stroke.pdf.

[2] V. L. Feigin, "Stroke epidemiology in the developing world," The Lancet, vol. 365, no. 9478, pp. 2160-2161, 2005.

[3] L. Liu, D. Wang, K. S. Lawrence Wong, and Y. Wang, "Stroke and stroke care in China: huge burden, significant workload, and a national priority," Stroke, vol. 42, no. 12, pp. 3651-3654, 2011.

[4] Y.-J. Wang, S.-M. Zhang, L. Zhang, and C.-X. Wang, "Chinese guidelines for the secondary prevention of ischemic stroke and transient ischemic attack 2010," CNS Neuroscience and Therapeutics, vol. 18, no. 2, pp. 93-101, 2012.

[5] M. C. F. Shamy and C. S. Jaigobin, "The complexities of acute stroke decision-making: a survey of neurologists," Neurology, vol. 81, no. 13, pp. 1130-1133, 2013.
[6] J.-H. Zhang, D. Wang, and M. Liu, "Overview of systematic reviews and meta-analyses of acupuncture for stroke," Neuroepidemiology, vol. 42, no. 1, pp. 50-58, 2014.

[7] P. Wu, E. Mills, D. Moher, and D. Seely, "Acupuncture in poststroke rehabilitation: a systematic review and meta-analysis of randomized trials," Stroke, vol. 41, no. 4, pp. e171-e179, 2010.

[8] V. Hopwood and G. T. Lewith, "Does acupuncture help stroke patients become more independent?" Journal of Alternative and Complementary Medicine, vol. 11, no. 1, pp. 175-177, 2005.

[9] S.-Y. Liu, C.-L. Hsieh, T.-S. Wei, P.-T. Liu, Y.-J. Chang, and T.-C. $\mathrm{Li}$, "Acupuncture stimulation improves balance function in stroke patients: a single-blinded cotrolled, randomized study," American Journal of Chinese Medicine, vol. 37, no. 3, pp. 483494, 2009.

[10] F. Tan, X. Wang, H. Q. Li et al., "A randomized controlled pilot study of the triple stimulation technique in the assessment of electroacupuncture for motor function recovery in patients with acute ischemic stroke," Evidence-Based Complementary and Alternative Medicine, vol. 2013, Article ID 431986, 8 pages, 2013.

[11] X. Zhang, B. Wu, K. Nie, Y. Jia, and J. Yu, "Effects of acupuncture on declined cerebral blood flow, impaired mitochondrial respiratory function and oxidative stress in multi-infarct dementia rats," Neurochemistry International, vol. 65, no. 1, pp. 23-29, 2014.

[12] A. M. K. Wong, T.-Y. Su, F.-T. Tang, P.-T. Cheng, and M.-Y. Liaw, "Clinical trial of electrical acupuncture on hemiplegic stroke patients," The American Journal of Physical Medicine and Rehabilitation, vol. 78, no. 2, pp. 117-122, 1999.

[13] Y.-S. An, S.-K. Moon, I.-K. Min, and D.-Y. Kim, "Changes in regional cerebral blood flow and glucose metabolism following electroacupuncture at LI 4 and LI 11 in normal volunteers.," Journal of Alternative and Complementary Medicine, vol. 15, no. 10, pp. 1075-1081, 2009.

[14] D. H. Geschwind, "DNA microarrays: translation of the genome from laboratory to clinic," The Lancet Neurology, vol. 2, no. 5, pp. 275-282, 2003.

[15] R. B. Stoughton, "Applications of DNA microarrays in biology," Annual Review of Biochemistry, vol. 74, pp. 53-82, 2005.

[16] K. Jin, X. O. Mao, M. W. Eshoo et al., "Microarray analysis of hippocampal gene expression in global cerebral ischemia," Annals of Neurology, vol. 50, no. 1, pp. 93-103, 2001.

[17] A. E. Baird, "Blood genomics in human stroke," Stroke, vol. 38, no. 2, pp. 694-698, 2007.

[18] V. L. R. Rao, K. K. Bowen, V. K. Dhodda et al., "Gene expression analysis of spontaneously hypertensive rat cerebral cortex following transient focal cerebral ischemia," Journal of Neurochemistry, vol. 83, no. 5, pp. 1072-1086, 2002.

[19] R. Schmidt-Kastner, B. Zhang, L. Belayev et al., "DNA microarray analysis of cortical gene expression during early recirculation after focal brain ischemia in rat," Molecular Brain Research, vol. 108, no. 1-2, pp. 81-93, 2002.

[20] A. Lu, Y. Tang, R. Ran, J. F. Clark, B. J. Aronow, and F. R. Sharp, "Genomics of the periinfarction cortex after focal cerebral ischemia," Journal of Cerebral Blood Flow and Metabolism, vol. 23, no. 7, pp. 786-810, 2003.

[21] M. P. Stenzel-Poore, S. L. Stevens, Z. Xiong et al., "Effect of ischaemic preconditioning on genomic response to cerebral 
ischaemia: similarity to neuroprotective strategies in hibernation and hypoxia-tolerant states," The Lancet, vol. 362, no. 9389, pp. 1028-1037, 2003.

[22] Y. Tang, H. Xu, X. Du et al., "Gene expression in blood changes rapidly in neutrophils and monocytes after ischemic stroke in humans: a microarray study," Journal of Cerebral Blood Flow and Metabolism, vol. 26, no. 8, pp. 1089-1102, 2006.

[23] Y. Tang, A. Lu, B. J. Aronow, K. R. Wagner, and F. R. Sharp, "Genomic responses of the brain to ischemic stroke, intracerebral haemorrhage, kainate seizures, hypoglycemia, and hypoxia," European Journal of Neuroscience, vol. 15, no. 12, pp. 1937-1952, 2002.

[24] E. Z. Longa, P. R. Weinstein, S. Carlson, and R. Cummins, "Reversible middle cerebral artery occlusion without craniectomy in rats," Stroke, vol. 20, no. 1, pp. 84-91, 1989.

[25] Z. R. Li, Experimental Acupuncture Science, China Press of Traditional Chinese Medicine, Beijing, China, 2003.

[26] S. Zausinger, E. Hungerhuber, A. Baethmann, H.-J. Reulen, and R. Schmid-Elsaesser, "Neurological impairment in rats after transient middle cerebral artery occlusion: a comparative study under various treatment paradigms," Brain Research, vol. 863, no. 1-2, pp. 94-105, 2000.

[27] M. Kanehisa and S. Goto, "KEGG: kyoto encyclopedia of genes and genomes," Nucleic Acids Research, vol. 28, no. 1, pp. 27-30, 2000 .

[28] K. J. Livak and T. D. Schmittgen, "Analysis of relative gene expression data using real-time quantitative PCR and the 2-(Delta Delta C(T)) method," Methods, vol. 25, no. 4, pp. 402-408, 2001.

[29] X.-C. M. Lu, A. J. Williams, C. Yao et al., "Microarray analysis of acute and delayed gene expression profile in rats after focal ischemic brain injury and reperfusion," Journal of Neuroscience Research, vol. 77, no. 6, pp. 843-857, 2004.

[30] M. C. Papadopoulos, R. G. Giffard, and B. A. Bell, "An introduction to the changes in gene expression that occur after cerebral ischaemia," British Journal of Neurosurgery, vol. 14, no. 4, pp. 305-312, 2000.

[31] M. Hori, T. Nakamachi, R. Rakwal et al., "Unraveling the ischemic brain transcriptome in a permanent middle cerebral artery occlusion mouse model by DNA microarray analysis," Disease Models \& Mechanisms, vol. 5, no. 2, pp. 270-283, 2012.

[32] F. R. Sharp, A. Lu, Y. Tang, and D. E. Millhorn, "Multiple molecular penumbras after focal cerebral ischemia," Journal of Cerebral Blood Flow and Metabolism, vol. 20, no. 7, pp. 1011-1032, 2000.

[33] M. Jäättelä, "Heat shock proteins as cellular lifeguards," Annals of Medicine, vol. 31, no. 4, pp. 261-271, 1999.

[34] J. Ramos-Cejudo, M. Gutiérrez-Fernández, B. RodríguezFrutos et al., "Spatial and temporal gene expression differences in core and periinfarct areas in experimental stroke: a microarray analysis," PLoS ONE, vol. 7, no. 12, Article ID e52121, 2012.

[35] M. Mirabelli-Badenier, V. Braunersreuther, G. L. Viviani et al., "CC and CXC chemokines are pivotal mediators of cerebral injury in ischaemic stroke," Thrombosis and Haemostasis, vol. 105, no. 3, pp. 409-420, 2011.

[36] M. Minami and M. Satoh, "Chemokines and their receptors in the brain: pathophysiological roles in ischemic brain injury," Life Sciences, vol. 74, no. 2-3, pp. 321-327, 2003.
[37] P. Wolinski and A. Glabinski, "Chemokines and neurodegeneration in the early stage of experimental ischemic stroke," Mediators of Inflammation, vol. 2013, Article ID 727189, 9 pages, 2013.

[38] X. S. Liu, Z. G. Zhang, R. L. Zhang, and et al, "Chemokine ligand 2 (CCL2) induces migration and differentiation of subventricular zone cells after stroke," Journal of Neuroscience Research, vol. 85 , no. 10 , pp. $2120-2125,2007$.

[39] K. Mizushima, Y. Miyamoto, F. Tsukahara, M. Hirai, Y. Sakaki, and T. Ito, "A novel G-protein-coupled receptor gene expressed in striatum," Genomics, vol. 69, no. 3, pp. 314-321, 2000.

[40] S. F. Logue, S. M. Grauer, J. Paulsen et al., "The orphan GPCR, GPR88, modulates function of the striatal dopamine system: a possible therapeutic target for psychiatric disorders?" Molecular and Cellular Neuroscience, vol. 42, no. 4, pp. 438-447, 2009.

[41] Z. Rahman, J. Schwarz, S. J. Gold et al., "RGS9 modulates dopamine signaling in the basal ganglia," Neuron, vol. 38, no. 6, pp. 941-952, 2003.

[42] V. Zachariou, D. Georgescu, N. Sanchez et al., "Essential role for RGS9 in opiate action," Proceedings of the National Academy of Sciences of the United States of America, vol. 100, no. 23, pp. 13656-13661, 2003.

[43] J. Blundell, C. V. Hoang, B. Potts, S. J. Gold, and C. M. Powell, "Motor coordination deficits in mice lacking RGS9," Brain Research, vol. 1190, no. 1, pp. 78-85, 2008.

[44] M. A. Moskowitz, E. H. Lo, and C. Iadecola, "The science of stroke: mechanisms in search of treatments," Neuron, vol. 67, no. 2, pp. 181-198, 2010.

[45] W.-C. Shyu, D. D. Liu, S.-Z. Lin et al., "Implantation of olfactory ensheathing cells promotes neuroplasticity in murine models of stroke," The Journal of Clinical Investigation, vol. 118, no. 7, pp. 2482-2495, 2008.

[46] X. Shi, Y. Kang, C. Chen et al., "A long-term observation of olfactory ensheathing cells transplantation to repair white matter and functional recovery in a focal ischemia model in rat," Brain Research, vol. 1317, pp. 257-267, 2010.

[47] V. Krishnan and E. J. Nestler, "The molecular neurobiology of depression," Nature, vol. 455, no. 7215, pp. 894-902, 2008.

[48] E. M. Whyte, B. H. Mulsant, J. Vanderbilt, H. H. Dodge, and M. Ganguli, "Depression after stroke: a prospective epidemiological study," Journal of the American Geriatrics Society, vol. 52, no. 5, pp. 774-778, 2004.

[49] R. G. Robinson and G. Spalletta, "Poststroke depression: a review," Canadian Journal of Psychiatry, vol. 55, no. 6, pp. 341349, 2010.

[50] G. Gainotti, G. Antonucci, C. Marra, and S. Paolucci, "Relation between depression after stroke, antidepressant therapy, and functional recovery," Journal of Neurology Neurosurgery and Psychiatry, vol. 71, no. 2, pp. 258-261, 2001.

[51] L. S. Williams, S. S. Ghose, and R. W. Swindle, "Depression and other mental health diagnoses increase mortality risk after ischemic stroke," The American Journal of Psychiatry, vol. 161, no. 6, pp. 1090-1095, 2004.

[52] T. Pohjasvaara, R. Vataja, A. Leppävuori, M. Kaste, and T. Erkinjuntti, "Depression is an independent predictor of poor long-term functional outcome post-stroke," European Journal of Neurology, vol. 8, no. 4, pp. 315-319, 2001. 
[53] E. Chemerinski, R. G. Robinson, and J. T. Kosier, "Improved recovery in activities of daily living associated with remission of poststroke depression," Stroke, vol. 32, no. 1, pp. 113-117, 2001.

[54] N. Mitsios, M. Saka, J. Krupinski et al., "A microarray study of gene and protein regulation in human and rat brain following middle cerebral artery occlusion," BMC Neuroscience, vol. 8, article 93, 2007. 


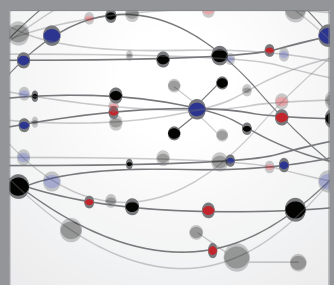

The Scientific World Journal
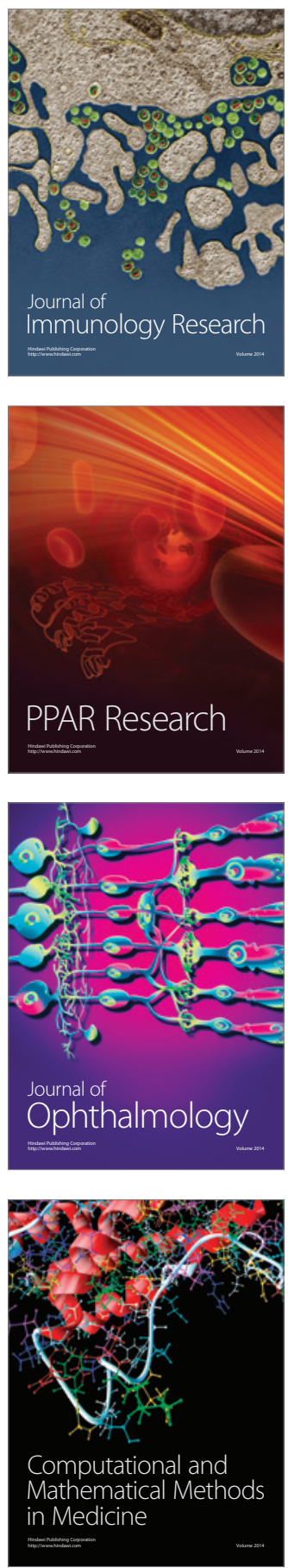

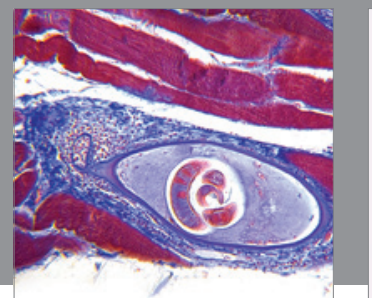

Gastroenterology

Research and Practice
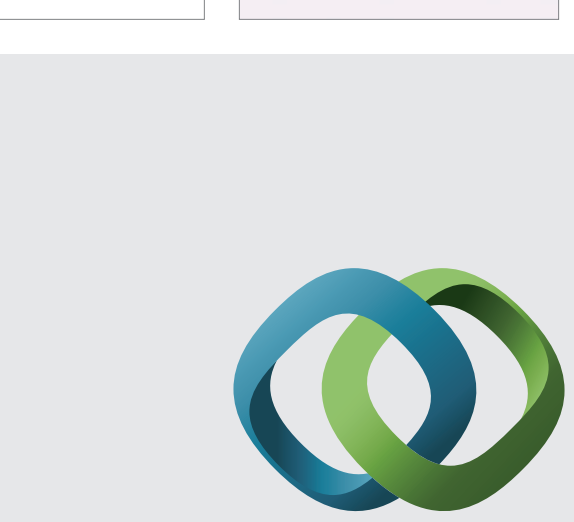

\section{Hindawi}

Submit your manuscripts at

http://www.hindawi.com
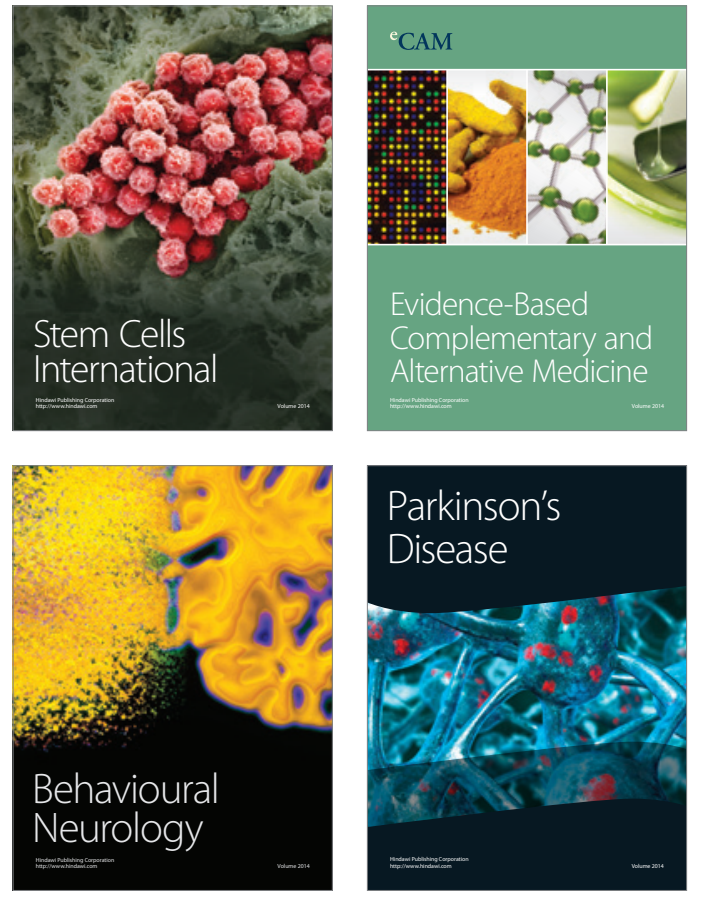
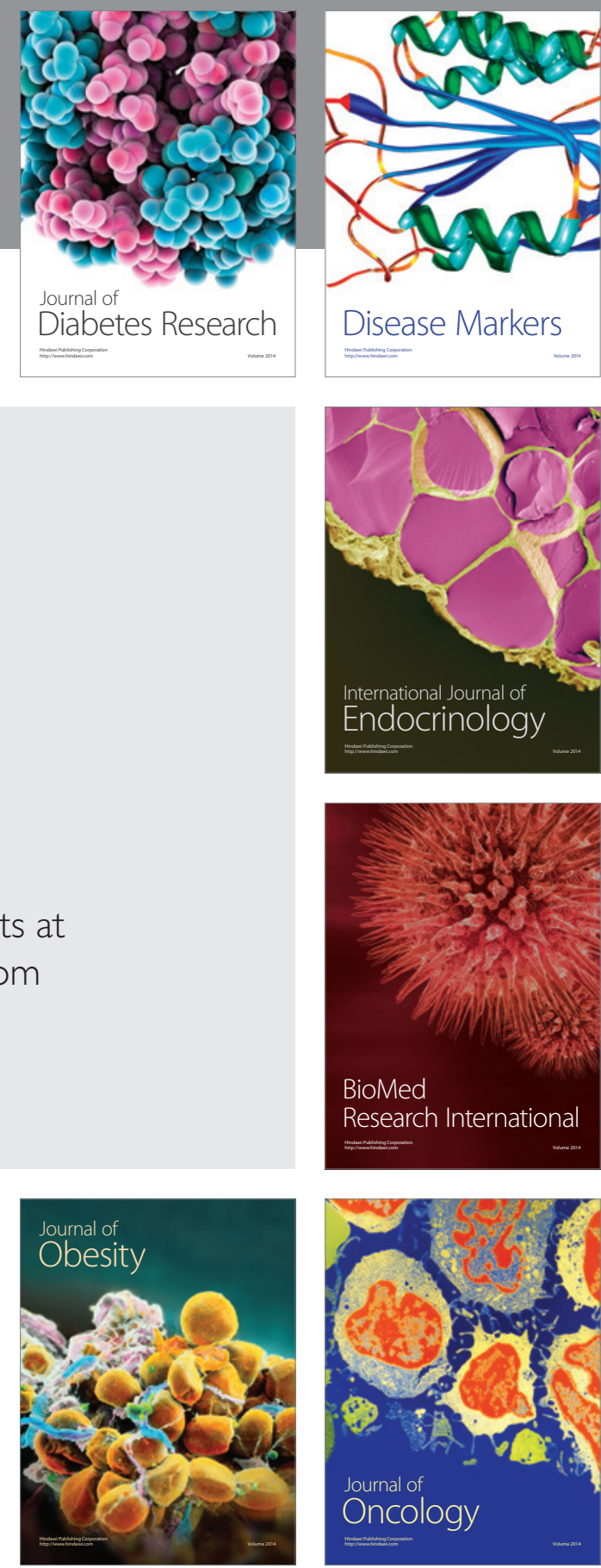

Disease Markers
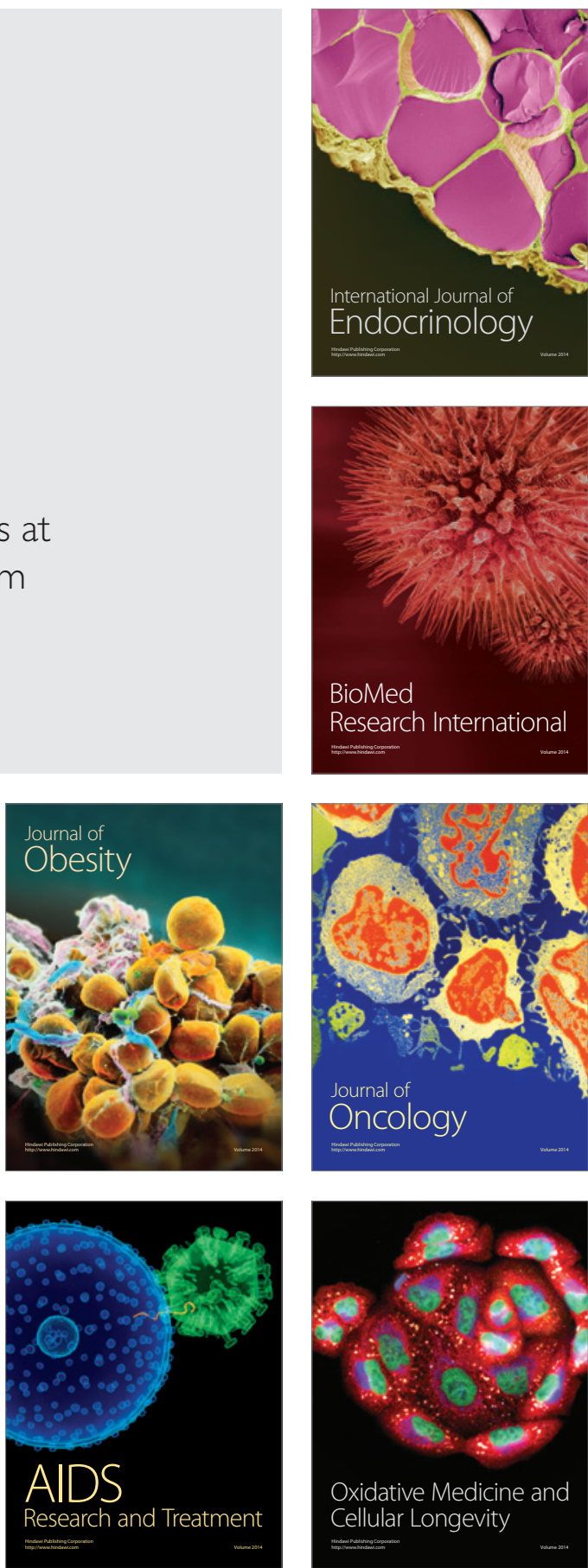